\title{
Compensating losses in negative-index metamaterials by optical parametric amplification
}

\author{
Alexander K. Popov \\ Department of Physics and Astronomy and Department of Chemistry, University of Wisconsin-Stevens Point, Stevens \\ Point, Wisconsin 54481-3897 \\ Vladimir M. Shalaev \\ School of Electrical and Computer Engineering, Purdue University, West Lafayette, Indiana 47907-2035
}

\begin{abstract}
Received February 6, 2006; revised April 17, 2006; accepted April 17, 2006; posted April 19, 2006 (Doc. ID 67834) Optical parametric amplification controlled by the auxiliary electromagnetic field enables transparency, amplification, and oscillation with no cavity in strongly absorbing negative-index metamaterials. The opposite directions of the wave vector and the Poynting vector in such materials result in extraordinary optical properties, including "backward" phase matching and the generation of entangled pairs of left- and right-handed counterpropagating photons. (C) 2006 Optical Society of America
\end{abstract}

OCIS code: 190.4410.

Metamaterials with a negative refractive index (NIMs), also referred to as left-handed metamaterials (LHMs), exhibit highly unusual electromagnetic properties and promise a variety of novel applications, particularly for nonlinear optical phenomena. As shown recently, NIMs that include structural elements with nonsymmetric current-voltage characteristics can possess a nonlinear magnetic response at optical frequencies ${ }^{1-3}$ and thus combine unprecedented linear and nonlinear electromagnetic properties. The opposite directions of the wave and Poynting vectors inherent for NIMs lead to extraordinary properties for basic optical processes in such metamaterials. The possibility of exact phase matching for waves with counterpropagating energy flows has been shown in Ref. 4 for the case of secondharmonic generation (SHG), when the fundamental wave is in the negative-index frequency domain while the $\mathrm{SH}$ wave is in the positive-index domain (PID). As seen from our considerations, phase matching of forward and backward waves is inherent for the nonlinear optics of NIMs. Important advantages of the interaction schemes involving counterdirected Poynting vectors in the process of optical parametric amplification for conventional right-handed materials (RHMs), also referred to as positive-index materials (PIMs), were discussed in early papers. ${ }^{5-7}$ However, in RHMs such schemes impose severe limitations on the frequencies of the coupled waves, because one of the waves must be in the far-infrared range in this case. ${ }^{5-7}$

Absorption is one of the largest problems that needs to be addressed to enable applications of NIMs. ${ }^{8,9}$ A transfer of the near-field image into the $\mathrm{SH}$ frequency domain, where absorption is typically much less, was proposed in Refs. 10 and 11 as a means to overcome dissipative losses and thus enable the NIM-based superlens. This Letter proposes an alternative means for compensating losses, by producing entire transparency amplification, or even lasing without a cavity in NIMs, with the aid of only one control auxiliary electromagnetic field. The underlying physical mechanism is based on optical parametric amplification (OPA), where a positive-index control field enables a loss-balancing OPA for a negativeindex signal wave. We also predict the possibility of the generation of entangled pairs of counterpropagating right- and left-handed photons.

We assume a negative-index wave at $\omega_{1}$ (signal; $n_{1}<0$ ) has a wave vector $\mathbf{k}_{1}$, which is directed along the $z$ axis, and an energy flow $\mathbf{S}_{1}$, which is directed against the $z$ axis [Fig. 1(a)]. The medium is illuminated by a higher-frequency wave traveling along the $z$ axis. The frequency of this radiation is $\omega_{3}$ and is in a PID $\left(n_{3}>0\right)$. The two coupled waves with codirected wave vectors $\mathbf{k}_{3}$ and $\mathbf{k}_{1}$ generate a differencefrequency idler at $\omega_{2}=\omega_{3}-\omega_{1}$, which is in a PID $\left(n_{2}\right.$ $>0$ ). The idle wave contributes back into the wave at $\omega_{1}$ through three-wave mixing (TWM) and thus enables OPA at $\omega_{1}$ by converting the energy of the pump field at $\omega_{3}$ into the signal. We note that, all three interacting waves have their wave vectors codirected along $z$, whereas the energy flow of the signal wave $\mathbf{S}_{1}$ is directed against $z$, and thus it is opposite to the energy flows of the two other waves $\mathbf{S}_{2}$ and $\mathbf{S}_{3}$. Such a coupling scheme is in contrast with the conventional

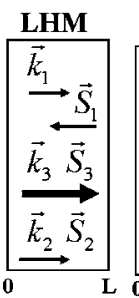

(a)

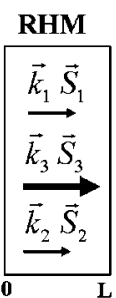

(b)

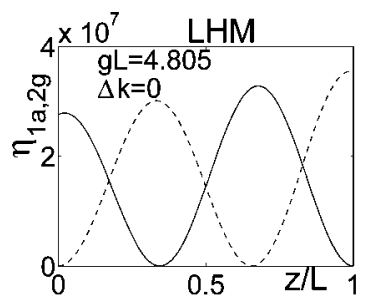

(c)

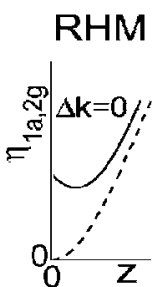

(d)
Fig. 1. OPA processes in NIMs and PIMs. Phase-matching schemes in (a) LHM and (b) RHM. The phase-matched amplification factor for the signal wave ( $\eta_{1 a}$, solid curve), and the conversion factor for the idler wave $\left(\eta_{2 g}\right.$, dashed curve) in (c) LHM and (d) RHM with absorption $\left(\alpha_{1} L=1, \alpha_{2} L\right.$ $=1 / 2$ ); (d) represents the dependence schematically. 
phase-matching scheme for OPA in RHMs [Fig. 1(b)]. This, in turn, results in different spatial intensity dependencies in LHMs and RHMs. Figure 1(c) displays the periodical oscillations along a NIM slab even at $\Delta k=0$, whereas exponential growth for both waves occurs in PIMs at large $z$ [Fig. 1(d)]. The noted results for NIMs are obtained from Eqs. (7) and (8); see below.

Optical magnetization plays a crucial role in NIMs. Following the method in Ref. 4, we assume that the nonlinear response is quadratic and associated with the magnetic component of the waves:

$$
H_{j}(z, t)=h_{j} \exp \left[i\left(k_{j} z-\omega_{j} t\right)\right]+\text { c.c. }
$$

$(j=1,2,3)$. The nonlinear magnetization at the signal and idler frequencies is given by

$$
M_{1,2}^{N L}=2 \chi_{\mathrm{eff}}^{(2)} h_{3} h_{2,1}^{*} \exp \left\{i\left[\left(k_{3}-k_{2,1}\right) z-\omega_{1,2} t\right]\right\} .
$$

Here $\omega_{2}=\omega_{3}-\omega_{1}, k_{j}=\left|n_{j}\right| \omega_{j} / c>0$, and $\chi_{\text {eff }}^{(2)}$ is the nonlinear susceptibility. Then, the slowly varying normalized amplitudes $a_{j}=\sqrt[4]{\epsilon_{j} / \mu_{j}} h_{j} / \sqrt{\omega_{j}}$ for the signal and idler waves can be written in the form

$$
\begin{gathered}
\mathrm{d} a_{1} / \mathrm{d} z=-i g a_{2}^{*} \exp (i \Delta k z)+\left(\alpha_{1} / 2\right) a_{1}, \\
\mathrm{~d} a_{2} / \mathrm{d} z=i g a_{1}^{*} \exp (i \Delta k z)-\left(\alpha_{2} / 2\right) a_{2},
\end{gathered}
$$

where $g=\left(\sqrt{\omega_{1} \omega_{2}} / \sqrt[4]{\epsilon_{1} \epsilon_{2} / \mu_{1} \mu_{2}}\right)(8 \pi / c) \chi^{(2)} h_{3}, \Delta k=k_{3}-k_{2}$ $-k_{1}$, and $\alpha_{j}$ are the absorption coefficients. The pump amplitude $h_{3}$ is assumed to be constant. The squared values of $a_{1,2}$ are proportional to the number of photons at the corresponding frequencies. We note the asymmetry in Eqs. (3) and (4), which is not the case for PIMs. The asymmetry reflects three fundamental differences in Eq. (3) as compared with a conventional difference-frequency generation (DFG) resulting from the TWM of copropagating waves in PIMs. First, the sign for the nonlinear polarization term is opposite to that in Eq. (4), which occurs because $\epsilon_{1}$ $<0$. Second, the opposite sign appears with $\alpha_{1}$ because the energy flow $\mathbf{S}_{1}$ is directed against the $z$ axis. Finally, the boundary conditions for $a_{1}$ are defined at the opposite side of the sample, as compared with $a_{2}$ and $h_{3}$, because the flows $\mathbf{S}_{1}$ and $\mathbf{S}_{2}$ are counterdirected. For $\alpha_{1}=\alpha_{2}=0$, one finds

$$
\begin{gathered}
\mathrm{d}\left[\left(S_{1 z} / \hbar \omega_{1}\right)-\left(S_{2 z} / \hbar \omega_{2}\right)\right] / \mathrm{d} z=0, \\
\mathrm{~d}\left[\sqrt{\mu_{1} / \epsilon_{1}}\left(h_{1}^{2} / \omega_{1}\right)+\sqrt{\mu_{2} / \epsilon_{2}}\left(h_{2}^{2} / \omega_{2}\right)\right] / \mathrm{d} z=0 .
\end{gathered}
$$

These equations represent the Manley-Rowe relations, which describe the creation of pairs of entangled counterpropagating photons $\hbar \omega_{1}$ and $\hbar \omega_{2}$. Equation (6) accounts for the opposite signs of the corresponding derivatives with respect to $z$; it predicts that the sum of the terms proportional to the squared amplitudes for the signal and idler will remain constant through the sample, which is in contrast with the requirement that the difference of such terms be constant in a PIM.
Taking into account the boundary conditions $a_{1}(L)=a_{1 L}$ and $a_{2}(0)=a_{20}$ (where $L$ is the slab thickness), the solutions to Eqs. (3) and (4) can be written as

$$
\begin{gathered}
a_{1}(z)=A_{1} \exp \left(\beta_{1}^{+} z\right)+A_{2} \exp \left[\left(\beta_{2}^{+} z\right)\right], \\
a_{2}^{*}(z)=\kappa_{1} A_{1} \exp \left(\beta_{1}^{-} z\right)+\kappa_{2} A_{2} \exp \left(\beta_{2}^{-} z\right),
\end{gathered}
$$

where

$$
\begin{gathered}
\beta_{1,2}^{ \pm}=\beta_{1,2} \pm(i \Delta k / 2), \quad \beta_{1,2}=\left(\alpha_{1}-\alpha_{2}\right) /(4) \pm i R, \\
A_{1,2}= \pm\left[a_{1 L} \kappa_{2,1}-a_{20}^{*} \exp \left(\beta_{2,1}^{+} L\right)\right] / D, \\
D=\kappa_{2} \exp \left(\beta_{1}^{+} L\right)-\kappa_{1} \exp \left(\beta_{2}^{+} L\right), \\
\kappa_{1,2}=( \pm R+i s) / g, \quad R=\sqrt{g^{2}-s^{2}}, \\
s=\left(\alpha_{1}+\alpha_{2}\right) /(4)-i(\Delta k / 2) .
\end{gathered}
$$

The amplification factor for the negative-index signal wave at $a_{20}=0$ is given by $\eta_{1 a}(z)=\left|a_{1}(z) / a_{1 L}\right|^{2}$. The corresponding conversion factor for the positive-index idler resulting from $\mathrm{DFG}$ is given by $\eta_{2 g}(z)$ $=\left|a_{2}(z)^{*} / a_{1 L}\right|^{2}$. Note that, a negative-index wave can be generated even when $a_{1 L}=0$; then the conversion factor for the signal is given by $\eta_{1 g}(z)=\left|a_{1}(z) / a_{20}^{*}\right|^{2}$.

Equations (7) and (8) predict unusual properties for the TWM process in a NIM, which are in sharp contrast with those in PIMs. Figures 2(a) and 2(b) show the oscillatory behavior of the amplification factor $\eta_{1 a}$ and the conversion efficiency for the idler $\eta_{2 g}$ at relatively large intensities of the control field $g L$ $=14.17$ for both $\Delta k=0$ and $\Delta k=\pi$. Note that, in contrast to PIMs, the oscillations occur even at $\Delta k=0$ [see also Fig. 1(c)]. This unusual behavior takes place because the signal and idler increase in opposite directions, and they are determined by the boundary conditions on the opposite sides of a NIM slab. As a result, the amplification threshold in NIMs appears for the product $g L$ rather than for $g$ as in PIMs. Figures 2(a) and 2(b) and 2(c)-2(e) also show strong dependence of amplification and the DFG efficiency on the phase matching for both larger $(g L=14.17)$ and smaller $(g L=1.82)$ magnitudes of the control field.

The comparison of Fig. 2(f) with Fig. 2(c) $(\Delta k=0$ in both figures) shows a strong resonance dependence of the DFG efficiency in NIMs on the parameter $g L$, indicating the necessity of fine tuning for the intensity of the control field. According to these figures, unless the pump intensity and phase matching are appropriately optimized, the maximum for the negativeindex signal may occur inside rather than at the output edge of the sample.

We also note that Figs. 2(a), 2(b), and 1(c) illustrate the generation of pairs of entangled right- and lefthanded photons propagating in opposite directions.

At $a_{20}=0$, the output value of $a_{10}$ is given by 


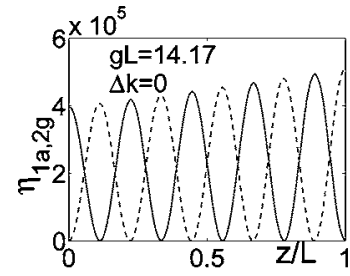

(a)

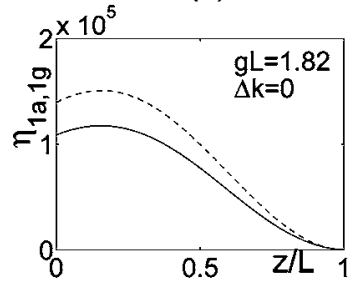

(c)

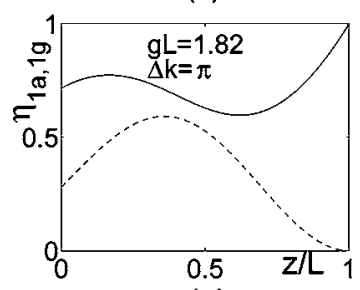

(e)

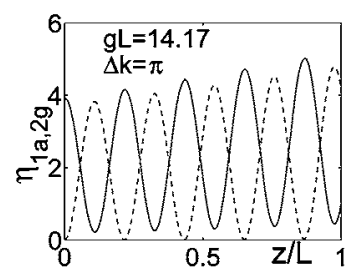

(b)

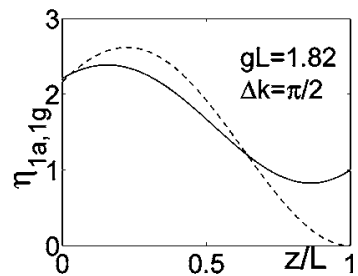

(d)

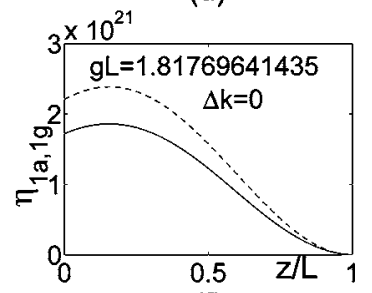

(f)
Fig. 2. Amplification factor for the negative-index signal $\left[\eta_{1 a}(z)=\left|a_{1} / a_{1 L}\right|^{2}\right.$; solid curves in (a)-(f)], the conversion factor for the signal $\left[\eta_{1 g}(z)=\left|a_{1} / a_{20}^{*}\right|^{2}\right.$; dashed curves in (c)-(f)]; and the conversion factor for the positive-index idler $\left[\eta_{2 g}(z)\left|a_{2} / a_{1 L}^{*}\right|^{2}\right.$; dashed curves in (a) and (b)] for various $\Delta k$ and $g L . \alpha_{1} L=1, \alpha_{2} L=1 / 2$.

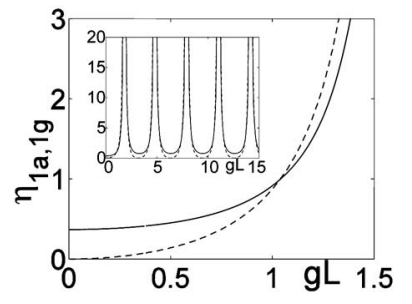

(a)

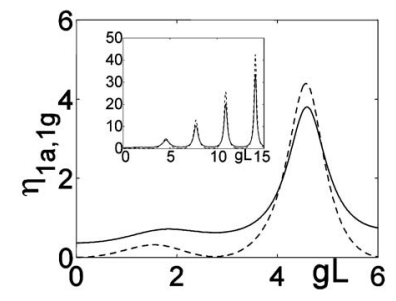

(b)
Fig. 3. Output amplification, $\eta_{1 a}$ (solid curve), and DFG conversion factor, $\eta_{1 g}$ (dashed curve) for the backward wave at $z=0$. (a) $\Delta k=0$. (b) $\Delta k L=\pi$. Insets, sets of periodical resonances for $\eta_{1 a, 1 g}$ versus $g L$. $\alpha_{1} L=1, \alpha_{2} L=1 / 2$.

$$
\frac{a_{1}(z=0)}{a_{1 L}}=\frac{\exp \left[-\left(\frac{\alpha_{1}-\alpha_{2}}{4}+i \frac{\Delta k}{2}\right) L\right]}{\cos R L+\left(\frac{\alpha_{1}+\alpha_{2}}{4 R}-i \frac{\Delta k}{2 R}\right) \sin R L} .
$$

Alternatively, at $a_{1 L}=0$, it is given by

$$
\frac{a_{1}(z=0)}{a_{20}^{*}}=\frac{-(g / R) \sin R L}{\cos R L+\left(\frac{\alpha_{1}+\alpha_{2}}{4 R}-i \frac{\Delta k}{2 R}\right) \sin R L}
$$

Equations (10) and (11) show that the output magnitude of the negative-index wave tends to infinity when the denominator approaches zero. This indicates the possibility of an oscillation with no cavity. For physics of cavityless oscillation, see Ref. 7 .

Figure 3 illustrates such resonance dependence on the intensity of the control field at $\omega_{3}$ (via the $g L$ parameter) for $\Delta k=0$ [Fig. 3(a)] and $\Delta k=\pi$ [Fig. 3(b)]. Figures 2 and 3 both show that the amplification for a phase-matched wave can reach many orders of magnitude in the maximums. Such OPA can not only compensate for absorption but even turn into optical parametric oscillations (OPOs) when the intensity of the control field reaches specific values given by a periodic set of the increasing numbers. The important advantage of the backward OPA and OPO in NIMs is distributed feedback, which enables oscillations without a cavity. In NIMs, each spatial point serves as a source for the generated wave in the reflected direction, whereas the phase velocities of all the three coupled waves are codirected.

In conclusion, we showed the feasibility of compensating losses in negative-index materials (NIMs) by OPA. In this process, the wave vectors of all three coupled waves are codirected, whereas the energy flow for the signal negative-index wave is counterdirected with respect to those for the pump and the idler waves. This process is characterized by properties that are in sharp contrast with those known for conventional nonlinear optical media. The possibility of OPOs without a cavity at frequencies where the refractive index is negative is also shown. Finally, OPA and OPO in NIMs enable the generation of pairs of entangled counterpropagating right- and left-handed photons.

The authors are grateful to V. V. Slabko for useful discussions and to S. A. Myslivets for help with numerical simulations. This work was supported in part by the National Science Foundation NIRT award ECS-0210445, the ARO-MURI Award, by U.S. Army Research Office grant W911NF-04-1-0350, and by the Defense Advanced Research Projects Agency under grant MDA 972-03-1-0020. Alexander Popov's e-mail address is apopov@uwsp.edu.

\section{References}

1. M. Lapine, M. Gorkunov, and K. H. Ringhofer, Phys. Rev. E 67, 065601 (2003).

2. A. A. Zharov, I. V. Shadrivov, and Yu. S. Kivshar, Phys. Rev. Lett. 91, 037401 (2003).

3. M. Lapine and M. Gorkunov, Phys. Rev. E 70, 66601 (2004)

4. I. V. Shadrivov, A. A. Zharov, and Yu. S. Kivshar, arXiv.org, http://arxiv.org/abs/physics/0506092.

5. S. E. Harris, Appl. Phys. Lett. 9, 114 (1966).

6. K. I. Volyak and A. S. Gorshkov, Radiotekh. Elektron. (Moscow) 18, 2075 (1973) (in Russian).

7. A. Yariv, Quantum Electronics, 2nd ed. (Wiley, 1975), Sec. 17.8.

8. K. J. Webb, M. Yang, D. W. Ward, and K. A. Nelson, Phys. Rev. E 70, 035602R (2004).

9. V. A. Podolskiy, N. A. Kuhta, and G. W. Milton, Appl. Phys. Lett. 87, 231113 (2005).

10. V. M. Agranovich, Y. R. Shen, R. H. Baughman, and A. A. Zakhidov, Phys. Rev. B 69, 165112 (2004).

11. A. A. Zharov, N. A. Zharova, I. V. Shadrivov, and Yu. S. Kivshar, Appl. Phys. Lett. 87, 091104-3 (2005). 